\title{
Comparison of naphthalene bioavailability determined by whole-cell biosensing and availability determined by extraction with Tenax
}

\author{
Stefanie Kohlmeier ${ }^{a}$, Matthew Mancuso ${ }^{b}$, Upul Deepthike ${ }^{b}$, Robin Tecon ${ }^{c}$, Jan Roelof van der Meer ${ }^{c}$, \\ Hauke Harms ${ }^{\mathrm{d}, *}$, Mona Wells ${ }^{\mathrm{b}, \mathrm{d}}$ \\ a Swiss Federal Institute of Technology (EPFL), Laboratory of Soil Science, CH-1015 Lausanne, Switzerland \\ ${ }^{\mathrm{b}}$ Tennessee Technological University, Department of Chemistry, Cookeville, TN 38505, USA \\ ${ }^{c}$ University of Lausanne, Department of Fundamental Microbiology, CH-1015 Lausanne, Switzerland \\ ${ }^{\mathrm{d}}$ UFZ, Helmholtz Centre for Environmental Research, Department of Environmental Microbiology, Permoserstrasse 15, D-04318 Leipzig-Halle, Germany
}

Whole-cell biosensing and mild extraction followed by chemical analysis quantify bioavailable naphthalene in sediment materials equally well.

\section{A R T I C L E I N F O}

\section{Article history:}

Received 3 September 2007

Received in revised form 30 May 2008

Accepted 1 June 2008

Available online $\mathrm{xxx}$

\section{Keywords:}

Bioavailability

Whole-cell biosensor

Contaminants

Pollution

Risk assessment

\begin{abstract}
A B S T R A C $T$
A rapid biological method for the determination of the bioavailability of naphthalene was developed and its value as an alternative to extraction-based chemical approaches demonstrated. Genetically engineered whole-cell biosensors are used to determine bioavailable naphthalene and their responses compared with results from Tenax extraction and chemical analysis. Results show a 1:1 correlation between biosensor results and chemical analyses for naphthalene-contaminated model materials and sediments, but the biosensor assay is much faster. This work demonstrates that biosensor technology can perform as well as standard chemical methods, though with some advantages including the inherent biological relevance of the response, rapid response time, and potential for field deployment. A survey of results from this work and the literature shows that bioavailability under non-equilibrium conditions nonetheless correlates well with $K_{\mathrm{oc}}$ or $K_{d}$. A rationale is provided wherein chemical resistance is speculated to be operative.
\end{abstract}

(c) 2008 Elsevier Ltd. All rights reserved.

\section{Introduction}

The bioavailability of environmental chemicals is an important determinant of toxicity and biodegradability. As the number of contaminated sites around the world rises, there will be an increasing need for site evaluation comprising the assessment of chemical risk and the chances to achieve natural or engineered biological cleanup. It is now accepted that bioavailability is a better indicator of risk than total chemical load (Alexander, 1995; Maddaloni, 2004; Peijnenburg et al., 2004; Pollumaa et al., 2004; Shuttleworth and Cerniglia, 1995; U.S. EPA, 1997, 1998; Wilson and Jones, 1993) and various chemical methods to quantify bioavailability have been developed. These chemical methods are typically more convenient than traditional ecotoxicological tests and often correlate with potential for biodegradation (Cornelissen et al., 1998; Volkering et al., 1998). In recent years, genetically engineered microorganisms have been used to detect target chemicals through induced expression of spectroscopically active reporter proteins (van der Meer et al., 2004). These organisms are

\footnotetext{
* Corresponding author. Tel.: +49 341235 1260; fax: +49 3412351351.

E-mail address: hauke.harms@ufz.de (H. Harms).
}

referred to as whole-cell biosensors or bioreporters. Biosensor bacteria make use of biological signalling chains coupled to luminescent, chromo- or fluorogenic proteins (van der Meer et al., 2004; Belkin, 2003; Kohler et al., 2000), such as shown in Fig. 1 for the strain used in this work. Biosensors theoretically enable quantitative measurements, as obtained with chemical methods, but with inherent biological relevance (van der Meer et al., 2004; Belkin, 2003; Harms et al., 2006).

Chemical approaches to bioavailability rely on extractions using mild solvents or chemical agents, membranes or other polymerbased devices (e.g. aqueous phase uptake by Tenax or XAD resins) (Cornelissen et al., 1998; Cuypers et al., 2000, 2002; Hawthorne et al., 2002; Krauss and Wilcke, 2001; Liste and Alexander, 2002; Macrae and Hall, 1998; Reid et al., 2000a,b; Volkering et al., 1998). If bioavailability is the availability to biota, then these chemical methods measure availability to chemical proxies, which are taken as representative to bioavailability. Using this chemical approach, a fraction $f$ of the chemical load is obtained that is empirically related to the rapidly released or labile contaminant fraction (Wells et al., 2004; Semple et al., 2004). Microorganisms capable of degrading organic contaminants represent a sink for this labile fraction $f$, but may display highly varied behaviour. Previous work has shown that microorganisms may influence bioavailability of 
A

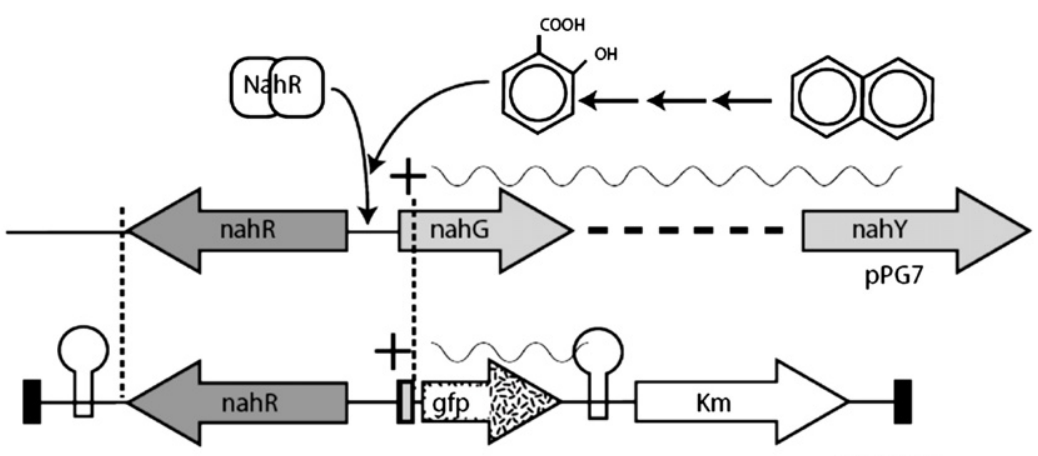

$\operatorname{mini}-\operatorname{Tn} 5$
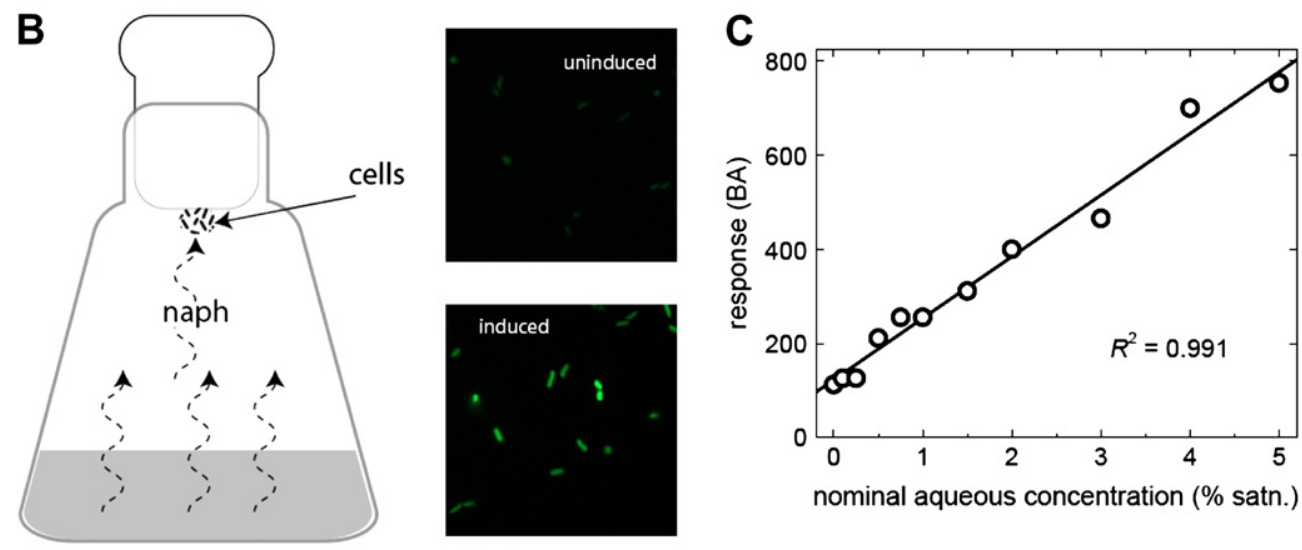

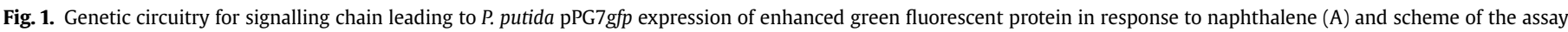

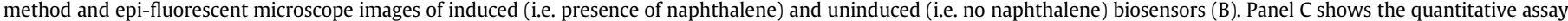

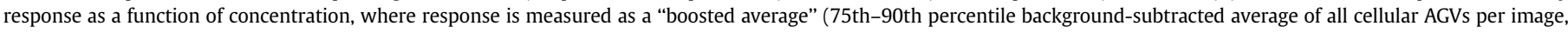
see Section 2).

environmental chemicals via formation of exudates such as biosurfactants and bioemulsifiers (Wells et al., 2004, 2005b). It is thus likely that biological variability affects the agreement between chemically determined $f$ and bioavailability.

As living organisms, biosensors may provide an inherently different approach to measuring bioavailability, and the substantive literature on chemical approaches to bioavailability provides the opportunity to evaluate the comparative performance of biosensors for the same purpose. A difficulty with such a comparison employing environmental samples is the need to exclude competing biodegradation during the chemical extraction; in previous work, we have performed chemical extraction either in sterile conditions or on biocided materials (Wells et al., 2005b). Since these conditions would preclude the use of living biosensors, we developed a biosensor-compatible assay for volatile analytes in which Pseudomonas putida strain pPG7gfp is separated by a headspace from a biocided sample containing naphthalene. Naphthalene, a polycyclic aromatic hydrocarbon (PAH) and constituent of coal tar, belongs to a class of widespread organic pollutants. Many bacterial species of Pseudomonas, Mycobacterium, Sphingomonas, Burkholderia and Ralstonia (Habe and Omori, 2003) degrade PAHs, and there is a substantial interest to optimize PAH bioremediation. However, biodegradation of PAHs in the environment is often limited by processes that reduce bioavailability (e.g. their strong tendency to sorb to organic matter (Bosma et al., 1997; Semple et al., 2003)). Hence, we used naphthalene as an analyte with environmental relevance that promised to improve our understanding of PAH bioavailability. Results reported here indicate excellent agreement between the biological and the chemical approach for samples having complex matrices that can exhibit strong effects on bioavailability.

\section{Materials and methods}

\subsection{Bacterial strain, media and growth conditions}

P. putida pPG7gfp expresses enhanced green fluorescent protein (egfp) in response to naphthalene (Fig. 1) as previously reported (Kohlmeier et al., 2007). The strain was continuously cultivated in chemostats (Werlen et al., 2004) using minimal medium (MM) buffered to $\mathrm{pH} 7$ (Wells, 2006) with $10 \mathrm{mM}$ D-glucose as a carbon source and $50 \mu \mathrm{g}$ of kanamycin per milliliter. Chemostats consisted of $1000-\mathrm{ml}$ glass flasks that were initially filled with $100 \mathrm{ml}$ medium and operated at a dilution rate of $0.06 \mathrm{~h}^{-1}$ at ambient temperature and constant stirring at $200 \mathrm{rpm}$. Pressurized air was used to aerate the medium. Each new chemostat was inoculated from a $-80^{\circ} \mathrm{C}$ glycerol stock, and after inoculation each chemostat culture was maintained for seven volume changes in order to reach steady state before biosensors were sampled. A sampling port allowed sampling with a 5-ml syringe. The culture was sampled every day to determine its optical density at $600 \mathrm{~nm}\left(\mathrm{OD}_{600}\right) . \mathrm{OD}_{600}$ values in the range between 1.8 and 2.1 indicated that cultures grew properly. The purity of the culture was checked every 2 days on LB plates without kanamycin.

\subsection{Naphthalene biosensor assays in the gas-phase}

The headspace assay (Fig. 1) was performed as previously described (Kohlmeier et al., 2007), save for addition of solids to the aqueous phase. In brief, glass test tubes with ground glass stoppers and a total volume of $16 \mathrm{ml}$ were filled with $5 \mathrm{~g}$ of solution containing $50 \mathrm{mg} / \mathrm{l} \mathrm{HgCl}$ as biocide. For introduction of solids into the assay, $0.2 \mathrm{~g}$ of the $5 \mathrm{~g}$ of solution was substituted with the solid of interest. The aqueous phase in each test tube was spiked with $20 \mu \mathrm{l}$ of a solution of naphthalene in dimethyl sulfoxide (DMSO) after the method of Werlen et al. (2004) to obtain final naphthalene concentrations ranging between 0.01 and $10 \mu \mathrm{M}$, and mixed well. Naphthalene-free DMSO was used to spike blank controls. After spiking the sample solutions, the test tubes were closed using the glass stoppers with $16 \mu$ l volume droplets containing biosensor cells of an $\mathrm{OD}_{600}$ of 1 deposited on the stopper tip. A small amount of water was added to the ground glass joint to ensure sealing (the lower aqueous diffusivity of naphthalene precluding gaseous diffusive loss during the time course of experiments). The cells were thus hanging from the inner side of the stopper, exposed to naphthalene only via the air and with no contact to the sample solution. The tubes were incubated at ambient temperature $\left(23^{\circ} \mathrm{C}\right)$ for $4 \mathrm{~h}$ 
without shaking. Then, the tubes were carefully opened and a micropipette was used to transfer the biosensors to microscope slides for analysis by epi-fluorescence microscopy (EFM). All samples were performed in triplicate. The time needed for EFM analysis effectively limited the number of samples. We and others are currently developing other detection methods (Wells, 2006).

\subsection{Biosensor immobilization to glass}

Glass slides were soaked in ethanol containing $5 \%$ potassium hydroxide $(\mathrm{w} / \mathrm{v})$ for $10 \mathrm{~min}$, rinsed with demineralized water and soaked in a solution of $0.05 \%$ poly L-lysine in water (Sigma-Aldrich). After $5 \mathrm{~min}$, the glass slides were taken out and rinsed again with demineralized water. Slides were then dried at $44{ }^{\circ} \mathrm{C}$ for $1 \mathrm{~h}$ and used directly for immobilization as described elsewhere (Jaspers et al., 2001).

\subsection{EFM analysis}

An Olympus BX-60 microscope with a BX-FLA fluorescence attachment was used for EFM. For each sample, three "replicate" analyses, consisting of three images, were taken with a Spot Insight QE camera (Diagnostic Instruments); exposure times varied from 250 to $2000 \mathrm{~ms}$. The number of bacteria in each image averaged $\sim 500$.

\subsection{Image and data analysis}

EFM images were analyzed by a Metamorph software routine described previously (Kohlmeier et al., 2007; Jaspers et al., 2001; Wells et al., 2005a). In brief, this involves image optimization for counting of fluorescent spots, followed by collection of statistics on each spot (size, shape, factor, average gray value or AGV). To exclude occasional random image artifacts, objects were filtered according to expected size and shape factor. To increase the sensitivity of our measurements, the backgroundsubtracted cellular AGVs of a more responsive subpopulation (the 75th-90th percentile subpopulation) not including the maximally responsive outliers was recorded.

\subsection{Tenax extractions}

First, $18 \mathrm{M} \Omega$ organics-purified water was added to 125-ml Erlenmeyer flasks, and, for assays utilizing solids, $0.2 \mathrm{~g}$ dry weight equivalent of solid sample, for a total of $100 \mathrm{~g}$ of solution or slurry. Flasks were spiked with $400 \mu$ DMSO solutions to obtain final desired naphthalene concentrations or with DMSO only for naphthalene-free controls. Four hours after spiking, a small amount of PAH-free 60/80 mesh Tenax (0.2 g, Supelco, Buchs, Switzerland; cleaned by sequential washing in water, methanol, hexane, and methanol prior to drying overnight at $80^{\circ} \mathrm{C}$ ) was added to each flask. The flasks were shaken at $130 \mathrm{rpm}$ and room temperature. Sampling after $4 \mathrm{~h}$ consisted of removing the Tenax using a small sterile stainless steel scoop. Removal was essentially quantitative since Tenax floats at the surface and sticks to the sides of the flask. The Tenax was extracted in a few $\mathrm{ml}$ of methanol and the extract analyzed by HPLC with UV detection on a Varian ProStar system equipped with a diode array detector operated in single-wavelength detection mode at $254 \mathrm{~nm}$ and using an 80:20 acetonitrile/water isocratic elution from a Vydac RP-C18 column. The accuracy of the HPLC analysis was verified using naphthalene control solutions (spiked) and standards.

\subsection{Sample suite}

The sample suite consisted of sediments and model materials. The model materials were kaolinite (Wards Scientific), organic-free by virtue of baking at $550{ }^{\circ} \mathrm{C}$ for $5 \mathrm{~h}$, and activated charcoal (Aldrich), pretreated using a method developed by Accardi-Dey and Gschwend (2002) such that the final material would be free of native PAHs, but retain the higher molecular weight aromatic backbone structure (the temperature sequence was a ramp at $15^{\circ} \mathrm{C} / \mathrm{min}$ to $300^{\circ} \mathrm{C}$, held for $5 \mathrm{~min}$, followed by a second ramp at $5{ }^{\circ} \mathrm{C} / \mathrm{min}$ to $375^{\circ} \mathrm{C}$, held for $24 \mathrm{~h}$, followed by cooling to room temperature). Sediment samples were from two Finnish Lakes (Lake Mekrijärvi and Lake Varparanta) and one lake in the Netherlands (Lake Ketelmeer).

\section{Results}

As with chemical analysis, the P. putida pPG7gfp headspace assay responds quantitatively to naphthalene over a linear range. The $R^{2}$ of $>0.99$ for the example given in Fig. $1 \mathrm{~B}$ is in line with standard chemical techniques used for naphthalene analysis (e.g. HPLC/ fluorescence detection, GCMS) thus giving confidence in the biosensor. To study how complex solid phases influence bioavailability, we incorporated model materials representing two extremes. Organic-free kaolinite was assumed to not affect availability of naphthalene in solution and activated charcoal to maximally reduce available naphthalene. The idea was thus to comprise all degrees of bioavailability possible in field samples. Fig. 2A shows
A

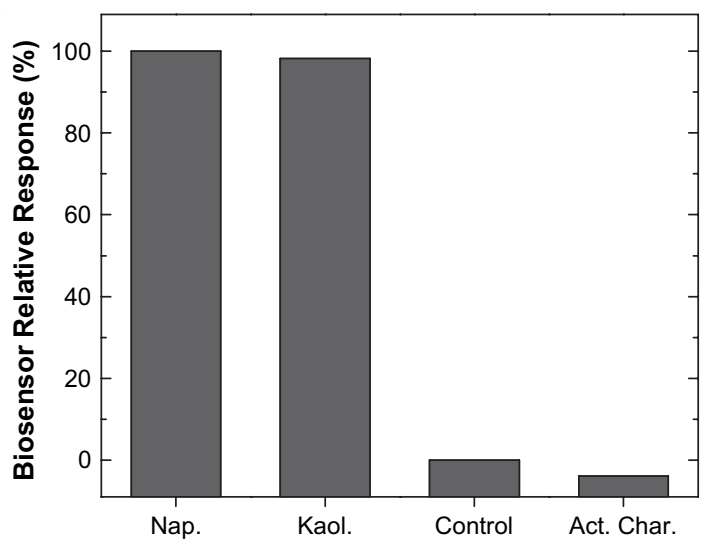

B

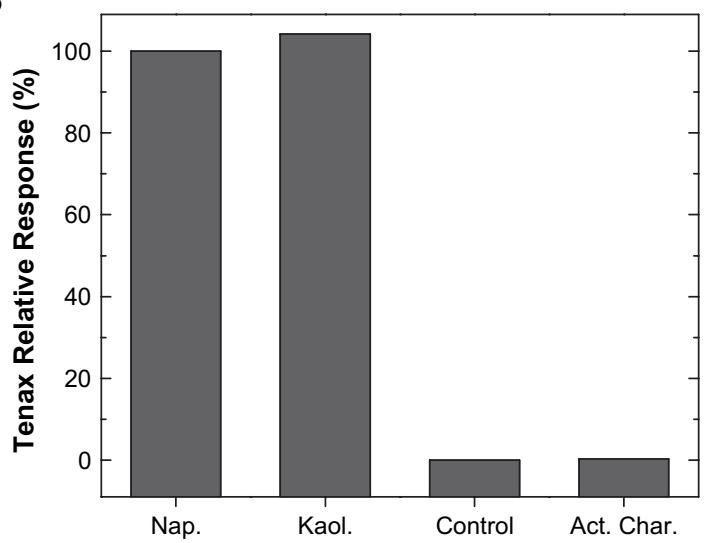

C

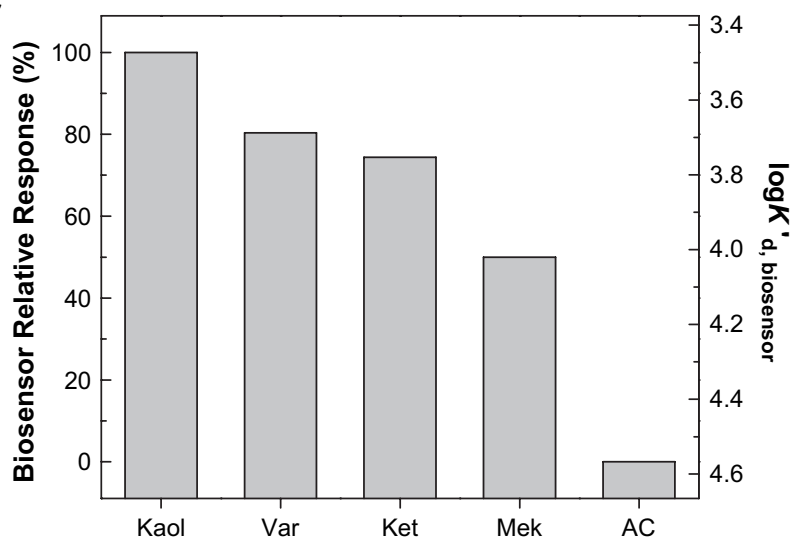

Fig. 2. (A) Results from biosensor experiments to validate model calibrant bioavailability using control solutions (spiked with naphthalene-free DMSO), naphthalene solutions (Nap.) and naphthalene solutions with model materials kaolinite (Kaol.) or activated charcoal (Act. Char.). Average relative standard deviation for replicate independent biosensor experiments was 9\%. (B) Results for Tenax extractions followed by chemical analysis for the identical experimental scheme as in panel A Tenax method varied from biosensor method as described in experimental details. Average relative standard deviation for replicate independent Tenax experiments was 7\%. (C) Results from post calibrant-validation biosensor bioavailability experiments on sediments using model materials calibration (Kaol=kaolinite, Var, Ket, and Mek = sediments from Lakes Varparanta, Ketelmeer, and Mekrijärvi, respectively, and AC=activated charcoal). Average relative standard deviation for triplicate analyses was $5 \%$. Righthand ordinate axis shows $K_{\text {biosensor }}^{\prime}$ using literature $K_{\mathrm{d}}^{\prime}$ values for kaolinite and activated charcoal as endpoints. For all experiments, solutions with naphthalene contained $0.3 \mathrm{mg} / \mathrm{l}$ (mid-linear range of biosensor response). 
the averaged results of multiple experiments validating our assumptions about the calibrants. The biosensor response was unaffected by kaolinite, whereas activated charcoal reduced biosensor responses even slightly below those in the naphthalene-free control. Since we routinely found this, we attributed it to the sensitivity of the gas-phase assay and the prevalence of trace quantities of naphthalene (or cross reacting compounds) in urban air. Fig. 2B serves as a secondary validation and shows that for experiments employing Tenax, the chemical proxy method chosen for comparison to biosensor results, naphthalene was also fully available in solutions containing kaolinite and unavailable in solutions containing activated charcoal.

Subsequent experiments included both calibrants and sediments that were previously characterized and used in bioavailability studies in the European Community project ABACUS. The sediments displayed representative extremes with regard to total organic carbon (TOC), black carbon (BC) content, and the ratio of TOC:BC. Lake Mekrijärvi sediment (Mek) had the highest TOC content $(24.3 \%)$ with only a small fraction of BC $(0.084 \%)$, whereas Lake Varparanta sediment (Var) displayed very little TOC $(0.5 \%)$, of which a significant portion was BC (0.024\%). Lake Ketelmeer sediment (Ket) had intermediate TOC (7.4\%), but the highest absolute BC $(0.72 \%)$. Results from biosensor assays on these sediments, relative to those obtained with the model calibrants are shown in Fig. $2 \mathrm{C}$ on the left-hand ordinate axis. Expressing response as a fraction facilitates comparison to $f$, the fraction available to Tenax. The right-hand ordinate axis expands over averaged naphthalene literature values of $\log K_{d}$ for kaolinite and various forms of black carbon (Bucheli and Gustafsson, 2000; Huang et al., 2005; Lee and Kim, 2002; Ko et al., 1998) as explained in the Section 4. The results in Fig. $2 \mathrm{C}$ were as expected: the model calibrants faithfully provided extremes as validated in Fig. $2 \mathrm{~A}$ and $\mathrm{B}$, between which lay the bioavailability of naphthalene for sediments chosen for compositional variation. The variation in bioavailability in Fig. $2 \mathrm{C}$ corresponded to variation in organic carbon content. A brief discussion of the effect of black carbon and black carbon models appears in Supplementary materials.

We performed independent Tenax experiments on the same suite of calibrants and samples used in Fig. $2 \mathrm{C}$ to quantify $f$. These results are plotted against the biosensor responses in Fig. 3. The good correlation $\left(R^{2}=0.95\right.$, which reduces to 0.85 when the data point representing the blank is removed) demonstrates that the

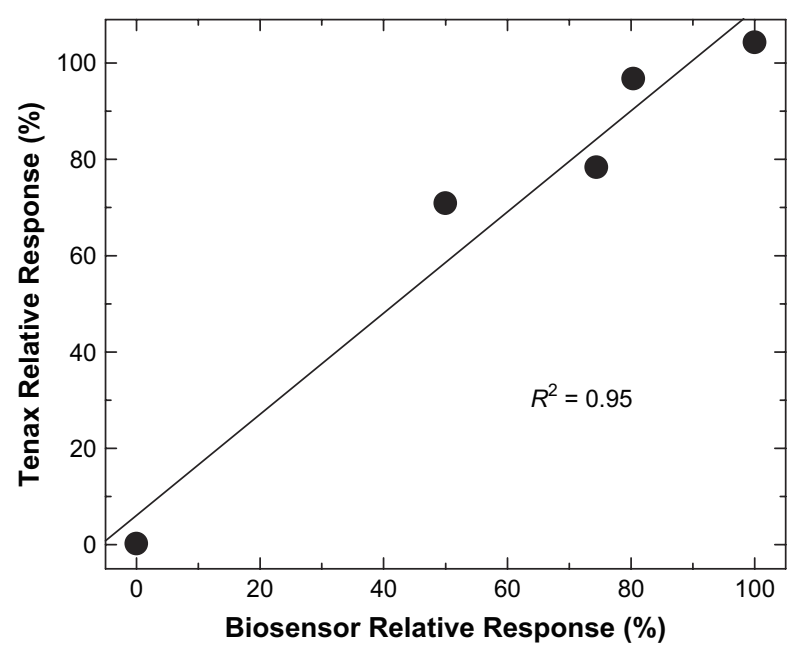

Fig. 3. Comparison of bioavailability and $f$ as obtained by Tenax extraction for independent experiments performed on sediments and model materials. The slope of the line obtained from linear regression is 1.05 . Average relative standard deviation for triplicate analyses is $7 \%$ for both experiments. headspace bioreporter assay can as well be used to quantify $f$, but with some advantages as pointed out below.

\section{Discussion}

Using bioavailability of pollutants in risk assessment has been discussed for some time. Already in 1989 the U.S. Environmental Protection Agency considered bioavailability as an element of risk assessment (Maddaloni, 2004). The implementation has been hindered by disagreement on definitions, and unavailability of tools to measure it and guidelines for data use in regulatory decisions (Alexander, 1995; Maddaloni, 2004; Peijnenburg et al., 2004; Pollumaa et al., 2004; Reid et al., 2000a; Semple et al., 2004; Chapman et al., 1998; Cura, 1998; Naidu et al., 2003). However, progress has been made, the development of biosensors being an essential component. For instance, the Microtox (Vibrio fischerii luminescence inhibition) assay is increasingly used in toxicity studies (Pollumaa et al., 2004; Belkin, 2003; Radix et al., 2000). The engineered strain used here falls into a class of bioreporters that responds highly selectively in a dose-dependent manner to one analyte or a narrow class of analytes (Belkin, 2003). The agreement between bioavailability, as measured by P. putida pPG7gfp and the kinetic parameter $f$ from Tenax extractions (Fig. 4) is encouraging. Previously, we examined experimental results wherein Tenax extractability of PAH sometimes correlated well with biodegradation and sometimes did not (Wells et al., 2005b). It is quite obvious that variations in the ability of microorganisms to mobilize sorbed substrates must lead to variable correlations with standardized chemical proxies. However, the spatial separation of our biosensors from the solid sample excludes biological mobilization of naphthalene beyond diffusion, thereby explaining the good correlation with the chemical proxy. The relative invariability of chemical extractability is explained by its frequent correlation to simple indicators of mass transport, of which aqueous solubility and effective diffusivity are such indicators (Wells et al., 2004, 2005b).

Results here indicate that using model calibrants, biosensor response is a good indicator of the value $f$, rapidly released or labile contaminant, employed in chemical extraction methods. This is conformal with the idea that $f$ is related to biodegradation. Using the empirical kinetic quantity $f$ to represent bioavailability obviates the need for equilibrium measurement that for sediments and soils may be impaired by reactions that approach equilibrium very slowly. However, the shift from an equilibrium-based to a kinetic approach is less consequent than apparent at first glance since there is a relationship between the kinetic quantity $f$ and apparent thermodynamic quantities such as $K_{\mathrm{oc}}$ and $K_{\mathrm{d}}$. This is illustrated in Fig. 4A-E, for both literature data and the present results. In each panel, the ordinate-axis values represent apparent thermodynamic quantities (either $K_{\mathrm{oc}}$ or $K_{\mathrm{d}}$ ) whereas the abscissa values are kinetic quantities ( $f$ from chemical extraction, biodegradation, or a value derived from biosensor response), and in each case there is a correlation between the two. In Fig. 4A and B experimental values of $f$ for various sediment and soil materials from the literature (Cornelissen et al., 1998; Hawthorne et al., 2002) are plotted against $K_{\text {oc. }}$ Data in Fig. $4 \mathrm{~A}$ result from XAD extraction of PAHs from the aqueous phase in the presence of contaminated soils (Hawthorne et al., 2002) and in Fig. 4B from Tenax extraction of contaminated Petrol Harbor sediment and a sample from a landfarming operation in Wemeldinge, both in the Netherlands (Cornelissen et al., 1998; Wells et al., 2005b). The data represent intra-sample variation for multiple PAHs, for which changes in $K_{\mathrm{oc}}$ can be large. However, given a single PAH, $K_{\mathrm{oc}}$ is constant. To reflect inter-sample variability in bioavailability, $K_{\mathrm{d}}$ may be used, and Fig. 4C illustrates the correlation of $K_{\mathrm{d}}$ with $f$ for literature data on naphthalene (Hawthorne et al., 2002).

Regarding biologically derived kinetic values, biodegradation experiments had been performed on some of the samples for which 
A

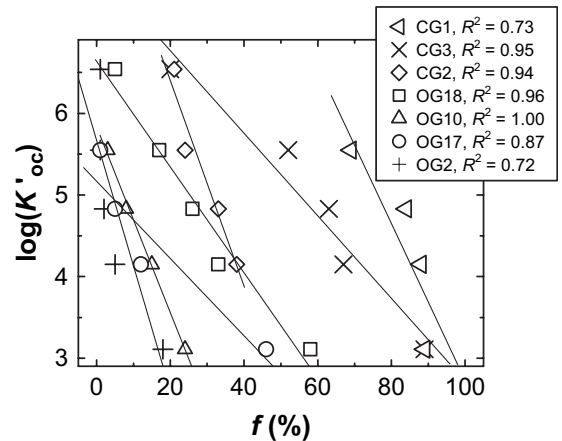

B
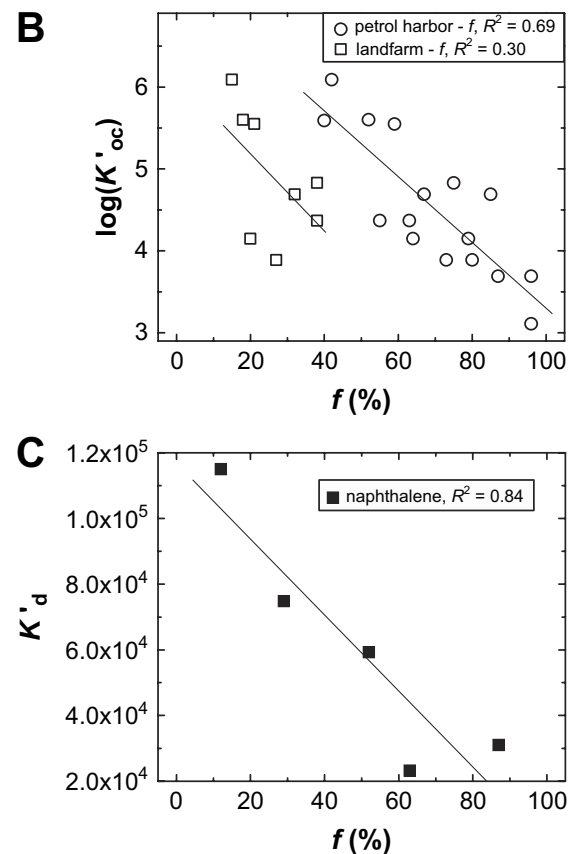

D
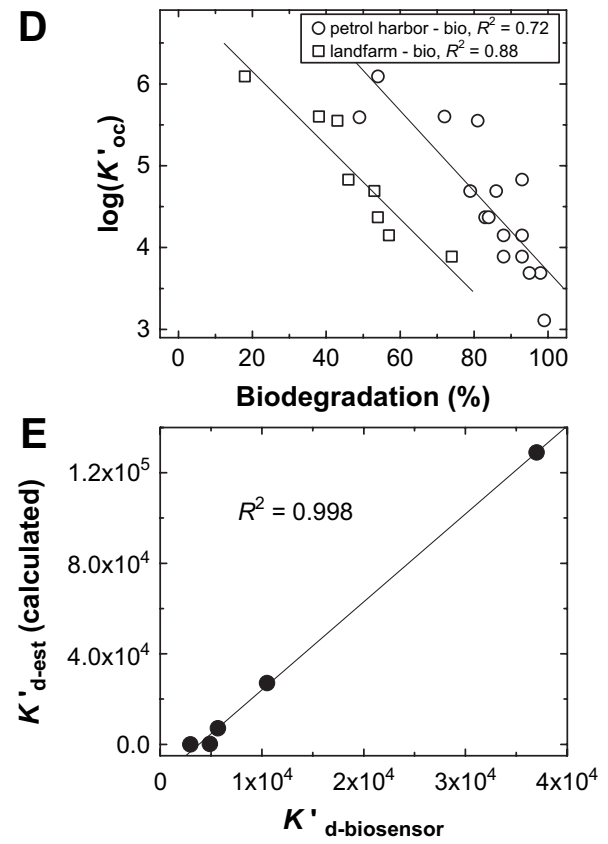

Tenax-based literature $f$ values are shown in Fig. 4B, and Fig. 4D shows that the correlation between biodegradation and $K_{\mathrm{oc}}$ $\left(R^{2}=0.72\right.$ and 0.88 for Petrol Harbor and the landfarm, respectively) is somewhat better than that between $f$ and $K_{\mathrm{oc}}$ (Fig. $4 \mathrm{~B}, R^{2}=0.69$ and 0.30 ). Correlations between biodegradation and $f$ are $R^{2}=0.68$ and 0.23 , respectively. To examine the present biosensor response similarly, in Fig. $4 \mathrm{E}$ the apparent thermodynamic quantity $K_{\mathrm{d}-\text { est. }}$ (i.e. estimated) is employed on the ordinate axis. It is calculated by dividing a literature $K_{\mathrm{oc}}$ by the fraction of organic carbon, $f_{\mathrm{oc}}$ of each sample. Values on the abscissa are for biosensor-inferred distribution coefficients, $K_{\text {biosensor }}$. They are linearly interpolated from calibrant endpoints, i.e. 0 and $100 \%$ bioavailability, and averaged $K_{\mathrm{d}}$ values for kaolinite and activated charcoal from various literature sources (Bucheli and Gustafsson, 2000; Huang et al., 2005; Lee and Kim, 2002; Ko et al., 1998). This refers to the right-hand ordinate axis in Fig. 2C wherein kaolinite, with highest bioavailability, corresponds to a low literature value of $K_{\mathrm{d}}$, and vice versa for activated charcoal. As such, $K_{\text {biosensor }}$ is a kinetic quantity rescaled, but importantly, a value entirely independently derived, the two independent quantities $\left(K_{\mathrm{d} \text {-est. }}\right.$ and $\left.K_{\text {biosensor }}\right)$ correlating at $R^{2}=0.998$ (the worst correlation with any four regressors is $R^{2}=0.94$ ).

That results from short-term experiments (in some cases hours, e.g. Tenax and biosensor experiments, Fig. $4 \mathrm{~A}-\mathrm{C}$ and $\mathrm{E}$ ) and other degradation experiments wherein equilibrium conditions are not expected to pertain (e.g. Fig. 4D), would correlate with $K_{\mathrm{oc}}$ or $K_{\mathrm{d}}$ has to our knowledge not been addressed in the manner we do here. While such correlations may not be immediately intuitive, we propose that it is a logical consequence of mass transport into and out of sediments and soils, the key to which is effective diffusivity, $D_{\text {eff. }}$ We previously demonstrated the relationship between effective diffusivity and release, including such ad hoc indicators as $f$, and found that $f$ is dependent on the value of $D_{\text {eff }}$ (Wells et al., 2004, $2005 b)$. However, $D_{\text {eff }}$ is in turn a function of the thermodynamic distribution coefficient, which represents chemical resistance to transport (in the expression $D_{\text {eff }}=\left(D_{\mathrm{H}_{2} \mathrm{O}} \varepsilon^{1 / 3}\right) /\left(1+(\rho / \varepsilon) K_{\mathrm{d}}\right)$, where $D_{\mathrm{H}_{2} \mathrm{O}}, \varepsilon$, and $\rho$ are free aqueous diffusivity, intraparticle porosity, and density, respectively). Hence it may even be that shortterm mass transport experiments could provide a means by which to infer information about chemical resistance, and certainly further studies are of interest to see if the calibration scheme we use here, employing model calibrants, will stand the test of extensibility to larger and more diverse sample sets.

Overall, results from this work indicate that biosensors perform as well as chemical extraction, but with some advantages, the most obvious of which is the inherent biological relevance of the biosensor response. Many chemical proxies for bioavailability require use of a biocide to prevent interference of native microorganisms with the chemical assay, potentially introducing an element of artificiality and potentially artefact into measurement. Additionally, biosensor assays can require as little as 500 biosensor cells (Kohlmeier et al., 2007), and such miniaturized assays are equally advantageous to both future development of field assays, and development of effectively zero sink assays (i.e. in chemical methods, a much larger quantity of chemical extractant is required for subsequent recovery, inevitably functioning as a sink, which

Fig. 4. (A) Literature results showing multianalyte relationships between $f$ and $K_{\mathrm{oc}}^{\prime}$ for XAD extractions of PAHs from contaminated soils (OG and CG stand for oil gas and coal-gas, respectively, see original literature in Hawthorne et al., 2002 for more information), and (B) Tenax extractions of PAHs from two contaminated sediments (data from Fig. 3 in Cornelissen et al., 1998). (C) Literature results showing single analyte inter-sample relationship between $f$ and $K_{\mathrm{d}}^{\prime}$ (Hawthorne et al., 2002). (D) For the data in panel $\mathrm{B}$, a stronger overall correlation is observed between biodegradation and $K_{\mathrm{oc}}^{\prime}$ than is observed for $f$ and $K_{\mathrm{oc}}^{\prime}$. (E) Relationship between $K_{\mathrm{d} \text {-est }}^{\prime}$ and $K_{\text {biosensor }}^{\prime}$ (obtained independently from each other). Values for $K_{\mathrm{d} \text {-est }}^{\prime}$ are calculated using $K_{\mathrm{oc}}^{\prime}$ and TOC results and values for $K_{\text {biosensor }}^{\prime}$ are inferred from interpolation using literature values for model calibrants and reporter response (i.e. an inherently kinetic quantity), see right-hand axis of Fig. $2 \mathrm{C}$ for graphical illustration of interpolation. 
then perturbs fluxes in any system under study). Finally, the biosensor appears to be faster - the Tenax method and the biosensor assay require a similar amount of preparation, and chemical extraction occurs over a similar period as biosensor induction for the strain used here (and most strains in use) - however, for instance with Tenax, after extraction with sediments, the analyte must be removed from the chemical extractant using a secondary solvent and then subjected to chemical analysis. In some cases a cleanup step would also need to be performed prior to chromatographic analysis to ensure proper chromatography and detection. Biosensor measurements produce results that are consistent with our previous work on the relation between bioavailability, rapid release and mass transport indicators such as effective diffusivity. Future work involves experiments designed to investigate bioavailability in more realistic conditions (i.e. permitting activity of native organisms by the absence of biocide).

\section{Acknowledgements}

We gratefully acknowledge Elena Rossel and Marie Sudki for valuable technical and administrative support, and for financial support, the Swiss Federal Office for Education and Science (Grant BBW01.0088), who funded the EC ABACUS project (EVK1-CT-200100101), and the 6th Framework European Programme, Contract 018391_FACEiT.

\section{Appendix A. Supplementary data}

Supplementary data associated with this article can be found, in the online version, at doi: 10.1016/j.envpol.2008.06.001.

\section{References}

Accardi-Dey, A., Gschwend, P.M., 2002. Assessing the combined roles of natural organic matter and black carbon as sorbents in sediments. Environ. Sci. Technol. $36,21-29$

Alexander, M., 1995. How toxic are toxic-chemicals in soil. Environ. Sci. Technol. 29, 2713-2717.

Belkin, S., 2003. Microbial whole-cell sensing systems of environmental pollutants. Curr. Opin. Microbiol. 6, 206-212.

Bosma, T.N.P., Middeldorp, P.J.M., Schraa, G., Zehnder, A.J.B., 1997. Mass transfer limitation of biotransformation: quantifying bioavailability. Environ. Sci. Technol. 31, 248-252.

Bucheli, T., Gustafsson, O., 2000. Quantification of the soot-water distribution coefficient of PAHs provides mechanistic basis for enhanced sorption observations. Environ. Sci. Technol. 34, 5144-5151.

Chapman, P.M., Wang, F., Janssen, C., Persoone, G., Allen, H.E., 1998. Ecotoxicology of metals in aquatic sediments: binding and release, bioavailability, risk assessment, and remediation. Can. J. Fish. Aquat. Sci. 55, 2221-2243.

Cornelissen, G., Rigterink, H., Ferdinandy, M.M.A., Van Noort, P.C.M., 1998. Rapidly desorbing fractions of PAHs in contaminated sediments as a predictor of the extent of bioremediation. Environ. Sci. Technol. 32, 966-970.

Cura, J.J., 1998. Ecological risk assessment. Water Environ. Res. 70, 968-971.

Cuypers, C., Grotenhuis, T., Joziasse, J., Rulkens, W., 2000. Rapid persulfate oxidation predicts PAH bioavailability in soils and sediments. Environ. Sci. Technol. 34, 2057-2063.

Cuypers, C., Pancras, T., Grotenhuis, T., Rulkens, W., 2002. The estimation of PAH bioavailability in contaminated sediments using hydroxypropyl-beta-cyclodextrin and Triton X-100 extraction techniques. Chemosphere 46, 1235-1245.

Habe, H., Omori, T., 2003. Genetics of polycyclic aromatic hydrocarbon metabolism in diverse aerobic bacteria. Biosci. Biotechnol. Biochem. 67, 225-243.

Harms, H., Wells, M., van der Meer, J.R., 2006. Whole-cell living biosensors: are they ready for environmental application? Appl. Microbiol. Biotech. 70, 273-280.

Hawthorne, S.B., Poppendieck, D.G., Grabanski, C.B., Loehr, R.C., 2002. Comparing PAH availability from manufactured gas plant soils and sediments with chemical and biological tests. 1. PAH release during water desorption and supercritical carbon dioxide extraction. Environ. Sci. Technol. 36, 4795-4803.

Huang, H.C., Lee, J.F., Chao, H.P., Yeh, P.W., Yang, Y.F., Liao, W.L., 2005. The influences of solid-phase organic constituents on the partition of aliphatic and aromatic organic contaminants. J. Colloid Interface Sci. 286, 127-133.
Jaspers, M.C., Meier, C., Zehnder, A.J., Harms, H., van der Meer, J.R., 2001. Measuring mass transfer processes of octane with the help of an alkS-alkB:gfp-tagged Escherichia coli. Environ. Microbiol. 3, 512-524.

Ko, S.-O., Schlautman, M.A., Carraway, E.R., 1998. Partitioning of hydrophobic organic compounds to sorbed surfactants. 1. Experimental studies. Environ. Sci. Technol. 32, 2769-2775.

Kohler, S., Belkin, S., Schmid, R.D., 2000. Reporter gene bioassays in environmental analysis. Fresenius J. Anal. Chem. 366, 769-779.

Kohlmeier, S., Mancuso, M., Tecon, R., Harms, H., van der Meer, J.R., Wells, M., 2007. Bioreporters: lux vs. gfp revisited and single cell response. Biosens. Bioelectron. 22, 1578-1585.

Krauss, M., Wilcke, W., 2001. Biomimetic extraction of PAHs and PCBs from soil with octadecyl-modified silica disks to predict their availability to earthworms. Environ. Sci. Technol. 35, 3931-3935.

Lee, S.Y., Kim, S.J., 2002. Adsorption of naphthalene by HDTMA modified kaolinite and halloysite. Appl. Clay Sci. 22, 55-63.

Liste, H.H., Alexander, M., 2002. Butanol extraction to predict bioavailability of PAHs in soil. Chemosphere 46, 1011-1017.

Maddaloni, M.A., 2004. Bioavailability of soil-borne chemicals: a regulatory perspective. Hum. Ecol. Risk Assess. 10, 657-663.

Macrae, J.D., Hall, K.J., 1998. Comparison of methods used to determine the availability of polycyclic aromatic hydrocarbons in marine sediment. Environ. Sci. Technol. 32, 3809-3815.

van der Meer, J.R., Tropel, D., Jaspers, M., 2004. Illuminating the detection chain of bacterial bioreporters. Environ. Microbiol. 6, 1005-1020.

Naidu, R., Bolan, N.S., Adriano, D.C., 2003. Bioavailability, toxicity, and risk relationships in ecosystems: the path ahead. In: Naidu, R., Gupta, V., Rogers, S.S. Kookana, R., Bolan, N., Adriano, D.C. (Eds.), Bioavailability, Toxicity and Risk Relationships in Ecosystems. Science Publishers, Enfield, New Hampshire, pp. 331-338.

Peijnenburg, W., Sneller, E., Sijm, D., Lijzen, J., Traas, T., Verbruggen, E., 2004. Implementation of bioavailability in standard setting and risk assessment. Environ. Sci. 11, 141-149.

Pollumaa, L., Kahru, A., Manusadzianas, L., 2004. Biotest- and chemistry-based hazard assessment of soils, sediments and solid wastes. J. Soils Sediments 4, 267-275.

Radix, P., Leonard, M., Papantoniou, C., Roman, G., Saouter, E., Gallotti-Schmitt, S. Thiebaud, H., Vasseur, P., 2000. Comparison of four chronic toxicity tests using algae, bacteria, and invertebrates assessed with sixteen chemicals. Ecotoxicol. Environ. Safe. 47, 186-194.

Reid, B.J., Jones, K.C., Semple, K.T., 2000a. Bioavailability of persistent organic pollutants in soils and sediments - a perspective on mechanisms, consequences and assessment. Environ. Pollut. 108, 103-112.

Reid, B.J., Stokes, J.D., Jones, K.C., Semple, K.T., 2000b. Nonexhaustive cyclodextrinbased extraction technique for the evaluation of PAH bioavailability. Environ. Sci. Technol. 34, 3174-3179.

Semple, K.T., Doick, K.J., Jones, K.C., Burauel, P., Craven, A., Harms, H., 2004. Defining bioavailability and bioaccessibility of contaminated soil and sediment is complicated. Environ. Sci. Technol. 38, 228A-231A.

Semple, K.T., Morris, A.W.J., Paton, G.I., 2003. Bioavailability of hydrophobic organic contaminants in soils: fundamental concepts and techniques for analysis. Eur. J. Soil Sci. 54, 809-818.

Shuttleworth, K.L., Cerniglia, C.E., 1995. Environmental aspects of PAH biodegradation. Appl. Biochem. Biotechnol. 54, 291-302.

U.S. EPA, 1997. The Incidence and Severity of Sediment Contamination in Surface Waters of the United States. EPA 823-R-97-006. U.S. EPA Office of Water, Washington, DC.

U.S. EPA, 1998. EPA's Contaminated Sediment Management Strategy. EPA 823-R-98001. U.S. EPA Office of Water, Washington, DC.

Volkering, F., Quist, J.J., Van Velsen, A.F.M., Thomassen, P.H.G., Olijve, M., 1998. A Rapid Method for Predicting the Residual Concentration After Biological Treatment of Oil-polluted Soil. Contaminated Soil '98. Thomas Telford, London, pp. 251-259.

Wells, M., 2006. Advances in optical detection strategies for reporter signal measurements. Curr. Opin. Biotech. 17, 28-33.

Wells, M., Gösch, M., Harms, H., van der Meer, J.R., 2005a. Response characteristics of arsenic-sensitive bioreporters expressing the gfp reporter gene. Microchim. Acta 151, 209-216.

Wells, M., Wick, L.Y., Harms, H., 2004. Perspectives on modeling the release of hydrophobic organic contaminants drawn from model polymer release systems. J. Mater. Chem. 14, 2461-2472.

Wells, M., Wick, L.Y., Harms, H., 2005b. A model polymer release system study of PAH bioaccessibility: the relationship between "rapid" release and bioaccessibility. Environ. Sci. Technol. 39, 1055-1063.

Werlen, C., Jaspers, M.C.M., van der Meer, J.R., 2004. Measurement of biologically available naphthalene in gas and aqueous phases by use of a Pseudomonas putida biosensor. Appl. Environ. Microb. 70, 43-51.

Wilson, S.C., Jones, K.C., 1993. Bioremediation of soil contaminated with polynuclear aromatic-hydrocarbons (PAHs) - a review. Environ. Pollut. 81, 229249. 